\title{
SARS-CoV-2-Induced Severe Immune Thrombocytopenic Purpura
}

\author{
Aurelien Gonze ${ }^{\mathrm{a}}$, Colin Hannesse ${ }^{\mathrm{a}}$, Nathalie Coppens ${ }^{\mathrm{a}}$, \\ Helene Vellemans ${ }^{\mathrm{b}}$, Gisele Maury ${ }^{\mathrm{a}, \mathrm{c}}$
}

\begin{abstract}
Hematologic changes are common in coronavirus infections, including lymphopenia and thrombocytopenia. Thrombocytopenia was frequent during the severe acute respiratory syndrome coronavirus (SARS-CoV) in 2002 - 2003. The mechanisms involved in platelet deficiency are unclear. Many viruses are known to trigger immune thrombocytopenic purpura (ITP), but the disease is rarely described in association with coronavirus. We describe the case of acute ITP associated with coronavirus disease 19 (COVID-19) in an 86-year-old woman. Intravenous gamma-globulin and corticosteroids were effective on platelet count evolution.
\end{abstract}

Keywords: Coronavirus; Thrombocytopenic purpura; Immune; Gamma-globulin; Corticosteroids; COVID-19

\section{Introduction}

Hematologic changes are common in coronavirus infections, including thrombocytopenia. Thrombocytopenia was frequent during the severe acute respiratory syndrome (SARS) linked to coronavirus in $2002-2003[1,2]$ and was considered as a severity criterion. The mechanisms involved in platelet deficiency are unclear. Many viruses are known to trigger immune thrombocytopenic purpura (ITP), but the disease is rarely described in association with coronavirus.

We aim to describe this association in the current context of severe acute respiratory syndrome coronavirus 2 (SARS-CoV-2) pandemic.

Manuscript submitted April 27, 2020, accepted May 5, 2020

Published online May 28, 2020

aPneumology Department, Clinique Saint Luc, Rue Saint Luc 8, 5004 Namur, Belgium

bHematology Department, Clinique Saint Luc, Rue Saint Luc 8, 5004 Namur, Belgium

${ }^{\mathrm{c} C o r r e s p o n d i n g ~ A u t h o r: ~ G i s e l e ~ M a u r y, ~ P n e u m o l o g y ~ D e p a r t m e n t, ~ C l i n i q u e ~}$ Saint Luc, Rue Saint Luc 8, B-5004 Namur, Belgium.

Email: Gisele.maury@slbo.be

doi: https://doi.org/10.14740/jmc3481

\section{Case Report}

An 86-year-old female patient presented with dyspnea and productive cough associated with a 3-day history of bleeding diathesis. The dyspnea was first felt on exertion 8 days ago, progressively increasing to at-rest dyspnea. Petechiae appeared on her limbs $24 \mathrm{~h}$ before admission. She was sent to the emergency department after occurrence of moderate intensity epistaxis associated with $<90 \% \mathrm{SpO}_{2}$ together with increasing dyspnea.

The patient's husband was hospitalized with the diagnosis of coronavirus disease 19 (COVID-19).

Patient's history was marked by aortic valve replacement and double coronary bypass grafting surgery 16 years earlier. Previous blood analyses were normal. There were no recent changes in her medications, consisting of aspirin, ramipril and simvastatin.

Upon arrival, the patient was awake, oriented, with blood pressure $172 / 75 \mathrm{~mm} \mathrm{Hg}$, heart rate $88 \mathrm{bpm}$, temperature 36.8 ${ }^{\circ} \mathrm{C}$ and $\mathrm{SpO}_{2} 94 \%$ without $\mathrm{O}_{2}$ supply.

Physical examination revealed signs of recent epistaxis as well as petechiae on the limbs (Fig. 1), lower back and on the oral mucosa without signs of oral bleeding. Macroscopic hematuria was noted.

Biologic results showed a slight hepatic cytolysis $(<2 \times$ normal values), a moderate inflammatory syndrome (C-reactive protein (CRP): $55 \mathrm{mg} / \mathrm{L} ; \mathrm{NR}:<5 \mathrm{mg} / \mathrm{L}$ ) without abnormal leucocytosis. Complete blood count showed severe thrombocytopenia $\left(7 \times 10^{9} / \mathrm{L}\right)$. Coagulation tests, folic acid and B12 vitamin were normal. Viral serologies for hepatitis B virus (HBV), hepatitis C virus (HCV), cytomegalovirus (CMV), Epstein-Barr virus (EBV), human immunodeficiency virus (HIV), parvovirus b19, herpes simplex virus (HSV) $1-2$, autoimmune markers (antinuclear antibodies (ANAs), antineutrophil cytoplasmic antibodies (ANCAs)) were all negative. On day 2 , lymphopenia (560 lymphocytes $/ \mathrm{mm}^{3}$ ) appeared.

Chest computed tomography (CT) showed multiple areas of bilateral ground-glass opacities, mostly peripheral, with an extensive degree of involvement (25-50\%). Respiratory viral panel polymerase chain reaction (PCR) was positive for SARS-CoV-2, negative for influenza A, B and respiratory syncytial virus (RSV). In the absence of contraindications, treatment with hydroxychloroquine $400 \mathrm{mg}$ bid in load dose, followed by $200 \mathrm{mg}$ bid for 5 days, was initiated according to our institutional treatment regimen. 


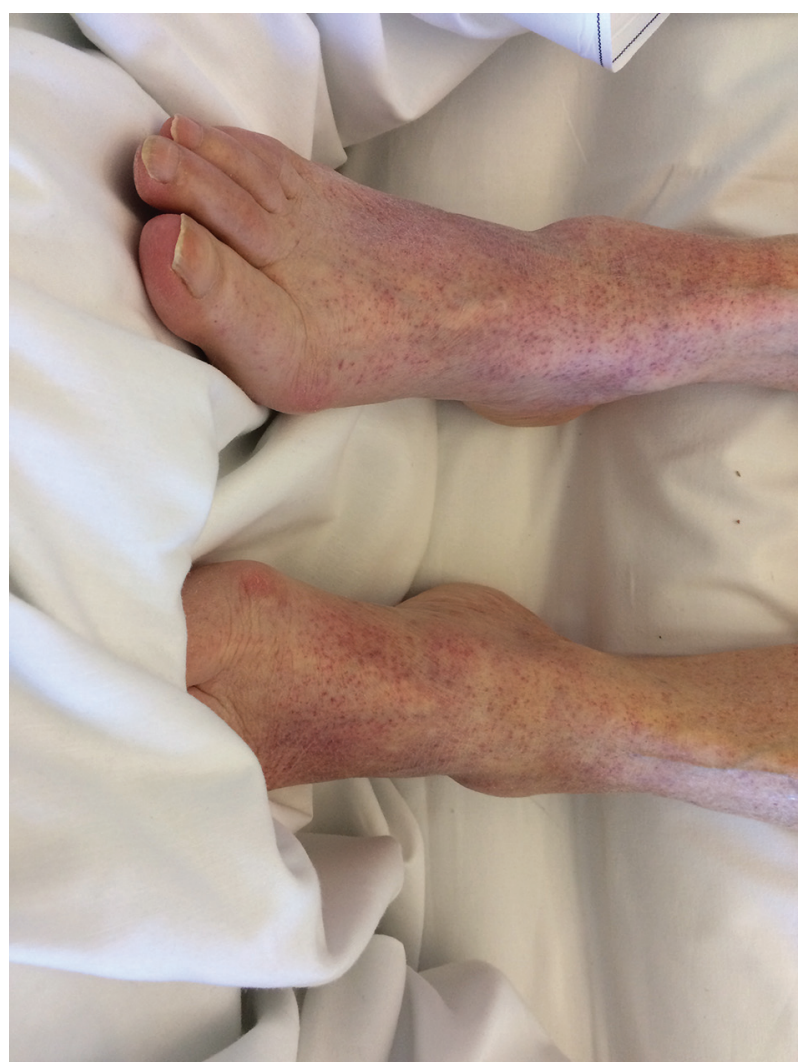

Figure 1. Purpuric lesions on the patient's lower limbs.

She underwent platelet transfusion on day 1 and on day 2 without significant increase in the circulating platelet count. Due to this suggestive clinical context, we decided not to perform bone marrow biopsy and considered the diagnosis of ITP.

On day 2, a corticosteroid treatment (CST) was started at a dose of $1 \mathrm{mg} / \mathrm{kg}$ of prednisone equivalent ( $80 \mathrm{mg}$ of intravenous (IV) methylprednisolone once a day) and IV gammaglobulin on day 3 at a dose of $400 \mathrm{mg} / \mathrm{kg} /$ day for a total of 5 days ( $25 \mathrm{~g}$ per dose administered).

On day 7, without any other platelet transfusion, platelet count rose to $144 \times 10^{9} / \mathrm{L}$. The patient left the hospital 2 weeks later.

\section{Discussion}

ITP is a rare disease, due to auto-immune mediated thrombocytopenia. Many viruses are known to trigger this disease. The authors describe the case of acute ITP associated with COVID-19 in an 86-year-old woman hospitalized for SARS. Dyspnea and bleeding diathesis were the reasons for consulting the emergency department.

During the 2002 - 2003 SARS-CoV, thrombocytopenia $\left(<150 \times 10^{9} / \mathrm{L}\right)$ occurred in $20 \%$ [1] to $55 \%$ [2] of patients. Thrombocytopenia was a severity criterion. Wong et al described the occurrence of thrombocytopenia $\left(<140 \times 10^{9} / \mathrm{L}\right)$ in $55 \%$ of patients followed by thrombocytosis $(49 \%)$ and iso- lated prolonged activated partial thromboplastin time (aPTT) $(63 \%)$. Only three patients had a severe form $\left(<50 \times 10^{9} / \mathrm{L}\right)$ [2]. When autopsy was performed, viral isolation for coronavirus showed negative results in the splenic, lymph node and bone marrow tissues which showed normal megakaryocytes. An immune cause is evoked for this thrombocytopenia. It is important to note that early CST was given as SARS standard treatment protocol.

Potential mechanisms of thrombocytopenia in SARS$\mathrm{CoV}$ include a direct effect of infection on megakaryocytes and platelets and/or immune damage of progenitor cells or platelets as well as low grade of disseminated intravascular coagulopathy [3]. The role of increased consumption in damaged lungs has been evoked [3].

A study performed on 41 patients suffering from COVID-19 pneumonia emerged in Wuhan showed thrombocytopenia at admission $\left(<100 \times 10^{9} / \mathrm{L}\right)$ in $5 \%$ [4].

In another recent study on 95 patients, platelet count $<100$ $\times 10^{9} / \mathrm{L}$ was present in $11.6 \%$ and $45.5 \%$ of them were severe cases [5].

Thrombocytopenia is important to consider in discriminating severe and non-severe coronavirus infections and is a predictive criterion of worsening of the disease in hospitalized patients with COVID-19 [6]. In Chinese hospitalized COVID-19 patients, first, thrombopenia is frequently seen but, in contrast, ITP is not described.

Recently, an early and acute ITP was described in a 65-year-old woman hospitalized for COVID-19 by Zulfiqar et al [7]. In this presentation, platelet count was normal at hospital admission and lower-extremity purpura concomitantly with isolated thrombocytopenia occurred on day 4. Previously, acute ITP associated with coronavirus HKU1 was described in a 24-year-old healthy man [8]. Clinical presentation was prolonged gum bleeding and diffuse rash skin on his extremities and abdomen with minor signs of respiratory infection 1 week before. As with our patient, clinical signs of the ITP were the reasons for consulting the emergency department. Considering that up to $18 \%$ of COVID-19 patients are asymptomatic, testing for SARS-CoV-2, either by PCR or serological test, should be considered in a patient presenting with a biological pattern evocative of an IPT. In these three ITP cases, IV immunoglobulin and CST were given with favorable platelet count evolution.

\section{Acknowledgments}

None to declare.

\section{Financial Disclosure}

None to declare.

\section{Conflict of Interest}

None to declare. 


\section{Informed Consent}

Not applicable.

\section{Author Contributions}

All the authors contributed to the editing of the manuscript. $\mathrm{AG}$ and $\mathrm{GM}$ wrote the manuscript.

\section{Data Availability}

The authors declare that data supporting the findings of this study are available within the article.

\section{References}

1. Tsang KW, Ho PL, Ooi GC, Yee WK, Wang T, ChanYeung M, Lam WK, et al. A cluster of cases of severe acute respiratory syndrome in Hong Kong. N Engl J Med. 2003;348(20):1977-1985.

2. Wong RS, Wu A, To KF, Lee N, Lam CW, Wong CK,
Chan PK, et al. Haematological manifestations in patients with severe acute respiratory syndrome: retrospective analysis. BMJ. 2003;326(7403):1358-1362.

3. Yang M, Ng MH, Li CK. Thrombocytopenia in patients with severe acute respiratory syndrome (review). Hematology. 2005;10(2):101-105.

4. Huang C, Wang Y, Li X, Ren L, Zhao J, Hu Y, Zhang $\mathrm{L}$, et al. Clinical features of patients infected with 2019 novel coronavirus in Wuhan, China. Lancet. 2020;395(10223):497-506.

5. Zhang G, Zhang J, Wang B, Zhu X, Wang Q, Qiu S. Analysis of clinical characteristics and laboratory findings of 95 cases of 2019 novel coronavirus pneumonia in Wuhan, China: a retrospective analysis. Respir Res. 2020;21(1):74.

6. Lippi G, Plebani B, Henry M. Thrombocytopenia is associated with severe coronavirus disease 2019 (COVID-19) infection: a meta-analysis. Clin Chim Acta. 2020.

7. Zulfiqar AA, Lorenzo-Villalba N, Hassler P, Andres E. Immune thrombocytopenic purpura in a patient with COVID-19. N Engl J Med. 2020;382(18):e43.

8. Magdi M, Rahil A. Severe immune thrombocytopenia complicated by intracerebral haemorrhage associated with coronavirus infection: a case report and literature review. Eur J Case Rep Intern Med. 2019;6(7):001155. 